\title{
Waste Collection Routing-Limited Multiple Landfills and Heterogeneous Fleet
}

\author{
Ana Maria Rodrigues \\ INESC TEC_-Technology and Science, Campus da FEUP, Rua Dr. Roberto Frias 378, 4200-465 Porto, Portugal
}

School of Accounting and Administration of Porto-Polytechnic Institute of Porto, Rua Jaime Lopes de Amorim, 4465-111 S.Mamede de Infesta, Portugal

\author{
José Soeiro Ferreira \\ INESC TEC_-Technology and Science, Campus da FEUP, Rua Dr. Roberto Frias 378, 4200-465 Porto, Portugal
}

Faculty of Engineering of the University of Porto, Campus da FEUP, Rua Dr. Roberto Frias, 4200-465 Porto, Portugal

This article deals with a real-life waste collection routing problem. To efficiently plan waste collection, large municipalities may be partitioned into convenient sectors and only then can routing problems be solved in each sector. Three diverse situations are described, resulting in three different new models. In the first situation, there is a single point of waste disposal from where the vehicles depart and to where they return. The vehicle fleet comprises three types of collection vehicles. In the second, the garage does not match any of the points of disposal. The vehicle is unique and the points of disposal (landfills or transfer stations) may have limitations in terms of the number of visits per day. In the third situation, disposal points are multiple (they do not coincide with the garage), they are limited in the number of visits, and the fleet is composed of two types of vehicles. Computational results based not only on instances adapted from the literature but also on real cases are presented and analyzed. In particular, the results also show the effectiveness of combining sectorization and routing to solve waste collection problems. @ 2015 Wiley Periodicals, Inc. NETWORKS, Vol. 65(2), 155-165 2015

Received October 2013; revised March 2014; accepted November 2014 Correspondence to: A. Maria Rodrigues; e-mail: amr@inesctec.pt (or) J. Soeiro Ferreira; e-mail: jsoeiro@inesctec.pt

Contract grant sponsor: National Funds through the FCT- Fundação para a Ciência e a Tecnologia (Portuguese Foundation for Science and Technology) within project "Project SEROW/ PTDC/ EGE-GES/ 121406/ 2010"

Contract grant sponsor: Project "NORTE-07-0124-FEDER-000057" is financed by the North Portugal Regional Operational Programme (ON.2 - O Novo Norte), under the National Strategic Reference Framework (NSRF), through the European Regional Development Fund (ERDF), and by national funds, through the Portuguese funding agency, Fundação para a Ciência e a Tecnologia (FCT)

DOI 10.1002/net.21597

Published online 2 February 2015 in Wiley Online Library (wileyonlinelibrary.com)

(C) 2015 Wiley Periodicals, Inc.
Keywords: waste collection; sectorization; mixed capacitated arc routing problem; heterogeneous fleet

\section{INTRODUCTION}

The collection and transportation of solid waste is a difficult and complicated problem of modern societies. Often it has to take into account different specifications and constraints.

The aim of this article is to present and deal with a reallife situation, based on the region of Monção, in the north of Portugal. The case reveals some specific characteristics which are not rare, at least in this country.

The size of municipalities is such that a prior sectorization is convenient. Multiple landfills and/or heterogeneous fleets are considered, since it is common for a company to use a heterogeneous fleet, which implies diverse costs, dimensions, capacities, and needs. Different vehicles may be required for different streets: for instance, some containers must be collected by a vehicle with a crane.

Another particular situation is related to the number and kind of disposal points. Here, two types of points are considered: landfills and transfer stations. Landfills could be used whenever a collection vehicle needs to be emptied. They have no limitation regarding the number of visits. Transfer stations are points that temporarily receive waste. Generally, transfer stations appear when the distance(s) between the center of collection and the landfill(s) exceed(s) $25 \mathrm{~km}$. After the disposal, other large vehicles are responsible for transporting waste from the transfer station to the landfill. However, sometimes transfer stations are small and are limited, which means that transfer stations cannot receive all 
the waste. In addition, more than one waste collection company may use the same place (landfill/transfer station) for waste disposal. Therefore, to deal with practical waste collection planning problems with multiple landfills (landfills and/or waste transfer stations), the limitations regarding the number of daily waste disposal visits should be incorporated in the optimization models. Regardless of the circuit's good location some stations may be small and therefore they only allow a limited number of visits for each company. Such circumstances arise, in particular, in the cases addressed in this work.

This article is organized as follows: section 2 briefly reviews the literature on capacitated arc routing problem (CARP) and waste collection. The real-life situation, waste collection in Monção, is described in section 3. After that, section 4 presents three models related to limited and multiple landfills and/or heterogeneous fleets. Section 5 provides the computational results based on different problems adapted from the literature and on real-life cases. Finally, section 6 draws some conclusions.

\section{CARPS AND WASTE COLLECTION}

Arc routing problems (ARP) are associated with determining a least cost traversal of the set (or subset) of edges, in a graph $G=(V, E)$, where $V$ is the set of vertices and $E$ is the set of edges. The graph can be undirected, directed (using the term arc instead of edge) or mixed (with edges and arcs). A cost $c_{i j} \geq 0$ is associated with each edge $(i, j), i \neq j$. If $(i, j) \notin E$, then $c_{i j}=\infty$. These problems appear in a large variety of practical contexts as referred in [12] such as: mail delivery, telephone book delivery, waste collection, street sweepers, salt gritting, street inspection for maintenance, meter reading, snow removal, school bus routing, internet routing, manufacturing cutting processes, or manufacturing printed circuit boards.

The CARP is a particular ARP and it appears when capacity restrictions are introduced in the closed paths of each vehicle. The CARP can be defined in an undirected, directed or mixed graph. Generally, a subset of edges must be served and a special vertex, called "depot," is often a landfill and a garage at the same time. A "depot" is not necessarily unique and, in the literature, "depot" and "garage" rarely correspond to different vertices. There exists a demand for each required edge. A fleet of vehicles (that are not necessarily identical) is available, and each vehicle has a finite capacity. The objective, as described in [27] and [28], is to find the minimum cost for a set of vehicle routes that must start and end at the depot, such that each required edge is serviced only once and the sum of demands of the serviced edges in each route does not exceed the vehicle capacity. The CARP is an NP-Hard problem and was introduced by [16]. A recent survey of the CARP and variants can be found in [39]. Capacitated restrictions are common in many problems. Some examples of these problems will be below along with references. The Capacitated Chinese Postman Problem, mentioned in [13], is a particular CARP where demands for all edges are positive. The Periodic
CARP, defined by [23] and [8], is an extension of the CARP to multiple periods. The CARP with Refill Points is a Location ARP with two different types of vehicles: the servicing vehicle and the refilling vehicle. Reference [1] presents a practical application for this problem. In the Sectorization ARP (SARP), presented in [31], the network is partitioned into a given number $K$ of sectors where the aim is to solve $K$ Mixed CARPs and to minimize the total duration of the trips made over all sectors. A special application is associated with waste collection in large urban areas. The Stochastic CARP, defined in [14], is a stochastic version of the CARP where demands on the arcs are random. The CARP with time dependent service costs, described by [38], is a variant of the CARP in which the cost of servicing some arcs depends on the time when the service begins. The extended CARP, in [25], addresses extensions, such as mixed multigraphs with edges, arcs, and parallel links, deadheading and collection costs per link, prohibited turns and turn penalties and an upper limit on the cost of any trip. In the CARP with Intermediate Facilities, vehicles may unload or replenish at intermediate facilities; examples of such situations can be found in [15] and [34]; waste collection with visits to dump sites or incinerators is another possible application. Other examples are CARP with Unit Demand, where all required edges present unit demand, see [6] and a multiobjective CARP, a version presented in [26]. An application of the latter version can be found in [24] where waste management companies are interested in both balancing the trips and minimizing their total duration. The Synchronized Arc and Node Routing Problem, described in [37], solves two routing problems simultaneously: a CARP with multivehicles and a node routing problem. Other general references recommended are: [9] and [3] to introduce ARP and capacitated problems.

A typical application of the general CARP is waste collection. There are many good examples in the literature that relate Waste Collection and Routing Problems. Reference [2] presents a constructive heuristic for waste collection in large cities from developing countries which also take environmental aspects into account. Two lower bounding methods and a three-phase heuristic for the CARP are presented by [30]. Reference [5] uses ant colony heuristics to solve an urban waste collection problem in the municipality of Sant Boi de Llobregat. A case study is described in [22]. Reference [31] aims to minimize the total duration of the circuits after dividing the street network into a certain number of sectors. Some authors, such as [21, 36, 33, or 4] deal with the problem of Waste Collection Routes as a Node Routing Problem. Most authors consider just one landfill, but [21], for instance, consider multiple disposal trips, where a variation of the vehicle routing problem with time windows and intermediate facilities is presented. Reference [11] deals with the situation of planning not only the location of the disposal site (landfills and transfer stations) but also the size of the landfills. In [10], a CARP with Mobile Depots is introduced. The problem is represented in an undirected graph with two types of vehicles: satellites and compactors. Compactors, with large dimension, are "moving depots" for satellites. 


\section{MONÇÃO WASTE COLLECTION}

The case presented is an outcome of the work of the authors in connection with waste collection problems in Monção. The municipality of Monção is a region in the north of Portugal with $220 \mathrm{~km}^{2}$ and a population of around 20,000 inhabitants. This county is a combination between rural and urban areas where the population is distributed in small villages around the 33 borough centers that constitute the municipality. This is a region with a strong component of emigrants and in the summer the population increases. As a consequence, the amount of waste produced increases as well. In the "more rural" regions, the waste must be collected two or three times a week (depending on the season), while in "more urban" regions waste must be collected every day. Throughout most of the year the company uses a large vehicle with a crane and, specially in the summer, the company uses a smaller to support the demand. In Monção, there are two disposal sites: the landfill of Valença and the transfer station of Messegães. The transfer station of Messegães also receives the waste of other municipalities. Inhabitants also use this transfer station to dump large objects such as old furniture. Generally, this transfer station only admits a single visit a day per vehicle. The municipality presents around 1,600 containers of different types, from simple trash bags to more modern and large containers. An estimation was made of the time a container is collected, which depends on its type. The average speed of the vehicle was also estimated $(30 \mathrm{~km} / \mathrm{h})$. The volume of each container was calculated considering the fact that, on average, containers are not completely full and vehicles have a system that compresses the waste collected. The fleet is composed of a vehicle with a crane with $16 \mathrm{~m}^{3}$ of capacity and a smaller $7 \mathrm{~m}^{3}$ vehicle. The vehicle with a crane takes $20 \mathrm{~min}$ to empty and the other takes $10 \mathrm{~min}$.

Consequently, and as previously mentioned, there are practical situations that involve various landfills some of which may impose limitations on the number of daily waste disposal visits. That may be due in particular to their size. Sometimes a landfill is not visited more than once a day by each vehicle.

To improve strategies for waste collection it is often convenient to partition the service area into sectors to know which part (sector) will be served each day and/or by which vehicles. It is important to highlight that the periodicity of the collection is not equal for all the boroughs in the county. This is what happens in Monção waste collection. The initial design of the sectors is accomplished by a new method inspired in Electromagnetism, as described in [35]. The method follows Coulomb's Law, which establishes a relation of force (attractive or repulsive) between electrically charged particles. Subsequently, each area/borough is represented by a particle whose location coincides with the centroid given by the distribution of containers in that borough. Each particle (borough) will create relations of attraction/repulsion with the others (two by two), which will be proportional to the "charges" (represented here by the amounts of waste to be collected in both boroughs) and inversely proportional to their distance (initially a Euclidean distance). Other authors have dealt with the sectorization problem, such as $[29,19,32$, or 31$]$.

\section{MODELS}

This section proposes three models, which are mixed CARPs with different attributes, related to heterogeneous fleet and limited multiple landfills. As mentioned in section 3, a sectorization phase takes place before the route planning process, also taking into account the periodicity of the waste collection.

\subsection{Model 1-Heterogeneous Fleet}

The Mixed Capacitated Arc Routing Problem with Heterogeneous Fleet reflects the situation in which a fleet, composed by three types of vehicles, leaves and returns to the same point every day. This place is a landfill and a garage (at the same time). Besides having different capacities, the vehicles may have various costs for traveling with or without collection (deadheading). Furthermore, some vehicles with collection cannot traverse some streets. The reasons are related to specific features of the streets and/or of the vehicles. Examples include the existence or non existence of a crane on the vehicle or the dimension of the street (narrow street).

Suppose there is a fleet with $P$ vehicles of three types $T_{1}$, $T_{2}$, and $T_{3}$, in amounts of $P_{1}, P_{2}$, and $P_{3}$, respectively.

These three sets form a partition of the set with $P$ (= $\left.P_{1}+P_{2}+P_{3}\right)$ vehicles.

$C$ is the set composed of $P$ ordered circuits. The first routes are from $T_{1}$ vehicles, then from $T_{2}$ vehicles, and finally from $T_{3}$ vehicles. Circuits $C_{1}, C_{2}$, and $C_{3}$ are defined as: $C_{1}=1,2, \ldots, P_{1} ; C_{2}=P_{1}+1, \ldots, P_{1}+P_{2}$ and $C_{3}=P_{1}+P_{2}+1, \ldots, P$, with $C=1,2, \ldots, P$.

Consider the graph $G=(V, A)$, where $V$ represents, as usual, the set of vertices and $A$ represents the set of links (edges and/or arcs). Vertex 1 represents the depot.

Required links represented by $R$ are divided into two groups, $A_{R}$ and $E_{R}$ which are, respectively, required arcs and edges.

The matrix $B=\left[b_{i j}\right]_{(i, j) \in A}$ defined below for each link $(i, j)$ represents the kind of access allowed. Three situations were considered: links (streets) that must be served only by $T_{3}$ vehicles, links that cannot be served by $T_{1}$ vehicles, and links, the most common situation, that do not have those limitations, which means that any kind of vehicle can collect waste in those streets.

$$
b_{i j}= \begin{cases}0, & \text { service to }(i, j) \text { is allowed to all vehicles } \\ 1, & \text { vehicles type } T_{1} \text { cannot serve }(i, j) \\ 2, & \text { only vehicles } T_{3} \text { can serve }(i, j)\end{cases}
$$

Consider $c_{i j}^{1}, c_{i j}^{2}$ and $c_{i j}^{3}$ as the costs to serve link $(i, j)$ with $T_{1}, T_{2}$, and $T_{3}$ vehicles, respectively. Following the same order $d_{i j}^{1}, d_{i j}^{2}$, and $d_{i j}^{3}$ represent the deadheading (traversing without collecting) costs related to link $(i, j)$. 
As previously mentioned, vehicles have different capacities. The capacities of $T_{1}, T_{2}$, and $T_{3}$ vehicles are, respectively, $Q_{1}, Q_{2}$, and $Q_{3}$.

The demand on each link $(i, j)$ is given by $q_{i j} \geq 0$. If $q_{i j}=0$, it means that link $(i, j)$ is an nonrequired link, that is, $(i, j) \in \bar{R}$ where $\bar{R}=A \backslash R$.

Associated with each type of vehicle, $T_{1}, T_{2}$, and $T_{3}$, is the cost of emptying $\lambda_{1}, \lambda_{2}$, and $\lambda_{3}$, respectively.

Decision variables are:

$$
x_{i j}^{p}= \begin{cases}1, & \text { if link }(i, j) \text { is served in circuit } p \\ 0, & \text { otherwise }\end{cases}
$$

$y_{i j}^{p}=$ number of times, that $\operatorname{link}(i, j)$, on circuit $p$, is traversed without collection.

$f_{i j}^{p}=$ the remaining demand in link $(i, j)$ of circuit $p$.

\section{Model 1}

Min

$$
\begin{aligned}
& \sum_{e=1}^{3}\left[\sum _ { p \in C _ { e } } \left(\sum_{(i, j) \in R} x_{i j}^{p} \cdot c_{i j}^{e}+\sum_{(i, j) \in A} y_{i j}^{p} \cdot d_{i j}^{e}\right.\right. \\
& \left.\left.+\lambda_{e}\left(\sum_{(i, 1) \in R} x_{i 1}^{p}+\sum_{(i, 1) \in A} y_{i 1}^{p}\right)\right)\right] \\
& \sum_{j:(i, j) \in A} y_{i j}^{p}+\sum_{j:(i, j) \in R} x_{i j}^{p}=\sum_{j:(j, i) \in A} y_{j i}^{p} \\
& +\sum_{j:(j, i) \in R} x_{j i}^{p}, \forall i \in V, p=1, \ldots, P \\
& \sum_{p \in C} x_{i j}^{p}=1, \forall(i, j) \in A_{R} \\
& \sum_{p \in C}\left(x_{i j}^{p}+x_{j i}^{p}\right)=1, \forall(i, j) \in E_{R} \\
& \sum_{j:(1, j) \in A} y_{1 j}^{p}+\sum_{j:(1, j) \in R} x_{1 j}^{p} \leq 1, p=1, \ldots, P \\
& \sum_{j:(1, j) \in A} f_{1 j}^{p}=\sum_{(i, j) \in R} q_{i j} x_{i j}^{p}, p=1, \ldots, P \\
& \sum_{i:(i, 1) \in A} f_{i 1}^{p}=\sum_{i:(i, 1) \in R} q_{i 1} x_{i 1}^{p}, p=1, \ldots, P \\
& \sum_{p \in C_{1}} x_{i j}^{p}=0, \forall(i, j) \in A_{R}: b_{i j}=1 \\
& \sum_{p \in C_{1}} x_{i j}^{p}+\sum_{p \in C_{2}} x_{i j}^{p}=0, \forall(i, j) \in A_{R}: b_{i j}=2 \\
& \sum_{p \in C_{1}}\left(x_{i j}^{p}+x_{j i}^{p}\right)=0, \forall(i, j) \in E_{R}: b_{i j}=1
\end{aligned}
$$$$
\sum_{j:(j, i) \in A} f_{j i}^{p}-\sum_{j:(i, j) \in A} f_{i j}^{p}=\sum_{j:(j, i) \in R} q_{j i} x_{j i}^{p}, i \in V \backslash\{1\}, p=1, \ldots, P
$$

$\sum_{p \in C_{1}}\left(x_{i j}^{p}+x_{j i}^{p}\right)+\sum_{p \in C_{2}}\left(x_{i j}^{p}+x_{j i}^{p}\right)=0, \forall(i, j) \in E_{R}: b_{i j}=2$

$f_{i j}^{p} \leq Q_{1}\left(y_{i j}^{p}+x_{i j}^{p}\right), \forall(i, j) \in A, p=1, \ldots, P_{1}$

$f_{i j}^{p} \leq Q_{2}\left(y_{i j}^{p}+x_{i j}^{p}\right), \forall(i, j) \in A, p=P_{1}+1, \ldots, P_{1}+P_{2}$

$f_{i j}^{p} \leq Q_{3}\left(y_{i j}^{p}+x_{i j}^{p}\right), \forall(i, j) \in A, p=P_{1}+P_{2}+1, \ldots, P$

$x_{i j}^{p} \in\{0,1\}, \forall(i, j) \in R, p=1, \ldots, P$

$f_{i j}^{p} \geq 0, \forall(i, j) \in A, p=1, \ldots, P$

$y_{i j}^{p} \geq 0$, integer, $\forall(i, j) \in A, p=1, \ldots, P$

Model 1 is a generalization of the model presented in [18].

Considering all vehicles, the objective function (M1-1) represents the sum of the three costs: collecting cost, deadheading cost, and dump cost. At each vertex $i \in V$ constraint (M1-2) guarantees contiguity. Each arc (edge) must be served once (M1-3)((M1-4)). Constraint (M1-5) imposes that the garage will not be "visited" more than once (to avoid dump costs). The remaining demand is updated (M1-7): if in trip $\mathrm{p}$ the arc $(j, i)$ is served then the remaining demand decreases $q_{j i}$ units. Constraints (M1-7) and (M1-8) are also flow conservation constraints. (M1-9)-(M1-12) indicate that some ars/edges cannot be served by some vehicle types. Upper bounds to guarantee capacity constraints are given by (M1-13)-(M1-15), which also ensure connectivity.

\subsection{Model 2-Limited Multiple Landfills}

The Mixed Capacitated Arc Routing Problem with Limited Multi-Landfills (MCARP-LML) is a mixed CARP with multiple landfills some of which allow a limited number of visits for waste disposal.

As explained before, there are practical situations involving various landfills, possibly imposing limitations on the number of daily waste disposal visits. Despite having a similar objective of Model 1, Model 2 minimizes the cost of trips between landfills and between landfills and the depot (garage), presenting several new characteristics: there is a single vehicle, the depot is not a disposal site, landfills, and/or transfer stations are not unique, and some disposal site may have a limited number of visits per day.

Before presenting the model, some definitions and notations are introduced. Consider a mixed graph $G=\left(V^{*}, L\right)$ with vertices $V^{*}$ and with links $L=A \cup E$, where $A$ and $E$ represent the set of arcs and the set of edges, respectively.

$V^{*}=V^{\prime} \cup V$ where $V^{\prime}=B \cup D . B$ is the set of vertices that correspond to landfills, $D$ is the depot (garage), and $V$ represents the ordinary vertices.

$R$ is the set of all required links: required $\operatorname{arcs} A_{R}$ and required edges $E_{R}$. $\tau$ represents the number of trips, a trip being a route between two vertices of $V^{\prime}=B \cup D$. 
$Q$ is the capacity of the vehicle.

$d_{i j}$ is the deadheading cost of link $(i, j) \in L$.

$c_{i j}>0$ is the cost of serving the required link $(i, j) \in R$ $\left(c_{i j}=0\right.$ if $\left.(i, j) \in \bar{R}\right)$.

$q_{i j}>0$ is the demand of link $(i, j) \in R\left(q_{i j}=0\right.$ if $\left.(i, j) \in \bar{R}\right)$

The first trip must start at the depot and the last must finish at the depot. When the vehicle leaves and returns to the depot it must be empty. The vehicle is emptied $\tau-1$ times at landfills. Each landfill $i$ has a maximum number of visits allowed $l_{i}$. The depot (or garage) is not a landfill.

Decision variables are:

$$
x_{i j}^{t}=\left\{\begin{array}{l}
1, \text { if edge } / \operatorname{arc}(i, j) \text { isserved by trip } t, \\
0, \text { otherwise }
\end{array}\right.
$$

$y_{i j}^{t}$ represents the number of times that arc/edge $(i, j)$ is deadheaded during trip $t$.

$z_{i j}^{t}$ is the quantity of waste collected during the trip $t$ after crossing arc/edge $(i, j)$. $\lambda$ is the cost of emptying.

The objective is to minimize the cost of the $\tau$ trips (M2-1).

\section{Model 2}

Min

$$
\sum_{t=1}^{\tau}\left(\sum_{(i, j) \in R} x_{i j}^{t} \cdot c_{i j}+\sum_{(i, j) \in L} y_{i j}^{t} \cdot d_{i j}+\lambda \sum_{b \in B:(i, b) \in L} y_{i b}^{t}\right)
$$

subject to

$$
\begin{aligned}
& \sum_{t=1}^{\tau}\left(\sum_{(i, j) \in L} y_{i j}^{t}+\sum_{(i, j) \in R} x_{i j}^{t}\right) \\
& =\sum_{t=1}^{\tau}\left(\sum_{(j, i) \in L} y_{j i}^{t}+\sum_{(j, i) \in R} x_{j i}^{t}\right), \quad \forall i \in V^{*} \\
& \sum_{(i, j) \in L} y_{i j}^{t}+\sum_{(i, j) \in R} x_{i j}^{t}=\sum_{(j, i) \in L} y_{j i}^{t} \\
& +\sum_{(j, i) \in R} x_{j i}^{t}, \quad \forall i \in V ; t=1, \ldots, \tau \\
& \sum_{t=1}^{\tau} x_{i j}^{t}=1, \quad \forall(i, j) \in A_{R} \\
& \sum_{t=1}^{\tau}\left(x_{i j}^{t}+x_{j i}^{t}\right)=1, \quad \forall(i, j) \in E_{R} \\
& \sum_{b \in V^{\prime}:(i, b) \in L} y_{i b}^{t}=1, \quad t=1, \ldots, \tau \\
& \sum_{b \in V^{\prime}:(b, j) \in L} y_{b j}^{t}=1, \quad t=1, \ldots, \tau \\
& \sum_{t=1}^{\tau} x_{i j}^{t}=0, \quad \forall(i, j) \in \bar{R}
\end{aligned}
$$

$$
\begin{aligned}
& x_{i j}^{\tau}=0, \quad \forall(i, j) \in R \\
& \sum_{(i, b) \in L} y_{i b}^{t}=\sum_{(b, j) \in L} y_{b j}^{t+1}, \quad t=1, \ldots, \tau-1 ; \forall b \in B
\end{aligned}
$$

$\sum_{t=1}^{\tau} \sum_{(b, j) \in L} y_{b j}^{t} \leq l_{b}, \quad \forall b \in B$

$\sum_{(i, b) \in L} y_{i b}^{t}+\sum_{(b, j) \in L} y_{b j}^{t} \leq 2, \quad t=1, \ldots, \tau ; \forall b \in V^{\prime}$

$\sum_{t=1}^{\tau} \sum_{b \in B} \sum_{(b, j) \in L} y_{b j}^{t}=\tau-1$

$\sum_{(i, j) \in L} z_{i j}^{t}-\sum_{(j, i) \in L} z_{j i}^{t}=\sum_{(i, j) \in R} q_{i j} \cdot x_{i j}^{t}, \quad \forall i \in V ; t=1, \ldots, \tau$

$\sum_{(i, b) \in L} z_{i b}^{t}=\sum_{(i, j) \in R} q_{i j} x_{i j}^{t}, \quad t=1, \ldots, \tau ; \forall b \in B$

$\sum_{(b, j) \in L} z_{b j}^{t}=0, \quad t=1, \ldots, \tau ; \forall b \in B$

$z_{i j}^{t} \leq Q\left(y_{i j}^{t}+x_{i j}^{t}\right), \quad \forall(i, j) \in L ; t=1, \ldots, \tau$

$x_{i j}^{t} \in\{0,1\}, \quad \forall(i, j) \in L ; t=1, \ldots, \tau$

$z_{i j}^{t} \geq 0, \quad \forall(i, j) \in L ; t=1, \ldots, \tau$

$y_{i j}^{t} \geq 0, \quad$ integer, $\forall(i, j) \in L ; t=1, \ldots, \tau$

The set of all trips must form a circuit (M2-2) and (M2-3). Each required arc (M2-4) and edge (M2-5) must be served just once. Each trip enters (M2-6) and leaves (M2-7) at some disposal site just once. A nonrequired edge is not served (M28). The garage is not a disposal site (M2-9). When a vehicle enters a disposal site for a certain trip, it must start the next trip at the same point (M2-10). The number of visits allowed for each disposal site is limited (M2-11). The quantity of waste collected in a trip is updated (M2-14) and (M2-15) after each service. After leaving a disposal site, the vehicle, must be empty (M2-16). The amount of waste collected cannot exceed the capacity of the vehicle (M2-17).

\subsection{Model 3-Heterogenous Fleet and Limited Multiple Landfills}

Model 3 combines the first two models. A MCARP-LML with two different vehicles: vehicle $T_{1}$ and vehicle $T_{2}$.

The notation used is similar to the one used in subsection 4.2 (Model 2): only the differences are presented next.

The set of required arcs, $A_{R}$, includes three disjoint subsets defined as: $A_{R 1}, A_{R 2}$, and $A_{R} \backslash\left(A_{R 1} \cup A_{R 1}\right)$ which, respectively, represent required arcs that must be served by vehicle $T_{1}$, required arcs that must be served by vehicle $T_{2}$, and required arcs that can be served by any vehicle. 
Similar restrictions are imposed on required edges, $E_{R}$ : $E_{R 1}, E_{R 2}$, and $E_{R} \backslash\left(E_{R 1} \cup E_{R 1}\right)$, which, similarly to the previous situation, limit the service of vehicle $T_{1}$ and vehicle $T_{2}$, respectively, and do not limit the access to any vehicles.

$W$ represents the number of trips for $T_{1}$ vehicles and $K$ is the number of trips for $T_{2}$ vehicles. It is important to remember that a trip is a route between two vertices of $V^{\prime}\left(V^{\prime}=B \cup D\right)$.

$Q_{1}$ and $Q_{2}$ identify the capacities of vehicles $T_{1}$ and $T_{2}$, respectively.

$d_{i j}^{1}\left(d_{i j}^{2}\right)$ corresponds to the deadheading cost for vehicle $T_{1}$ $\left(T_{2}\right)$ passing through the link $(i, j) \in L . c_{i j}^{1}>0$ and $c_{i j}^{2}>0$ are the costs of traversing with collection the link $(i, j) \in R$ $\left(c_{i j}=0\right.$ if $\left.(i, j) \in \bar{R}\right)$ using vehicle $T_{1}$ or $T_{2}$, respectively.

$\mathrm{q}_{i j}>0$ is the demand on link $(i, j) \in R\left(q_{i j}=0\right.$ if $\left.(i, j) \in \bar{R}\right)$.

The first trip of each vehicle must begin at the garage to where the vehicles must return at the end. Vehicles must be empty when they leave and when they return to the garage. In a working day, vehicle $T_{1}$ must empty $W-1$ times and vehicle $T_{2}, K-1$ times. Each disposal point $i$ has a maximum number of visits $l_{i}$ and the garage is not a disposal site. The number of visits to a landfill/transfer station is independent of the type of vehicle.

Decision variables $x_{i j b}^{t}, y_{i j}^{t}$, and $z_{i j}^{t}$ are introduced in subsection 4.2 .

The objective is to minimize the cost of $W+K(=\tau)$ trips. $\lambda_{b}^{1}$ and $\lambda_{b}^{2}$ represent the emptying costs at disposal site $b$ when the disposal is made by vehicle $T_{1}$ or vehicle $T_{2}$, respectively. As in previous models, required arcs/edges are not incident on the vertices that represent disposal sites.

\section{Model 3}

Min

$$
\begin{aligned}
& \sum_{w=1}^{W}\left(\sum_{(i, j) \in R} x_{i j}^{w} \cdot c_{i j}^{1}+\sum_{(i, j) \in L} y_{i j}^{w} \cdot d_{i j}^{1}+\sum_{b \in B:(i, b) \in L} \lambda_{b}^{1} \cdot y_{i b}^{w}\right)+ \\
& \sum_{k=W+1}^{\tau}\left(\sum_{(i, j) \in R} x_{i j}^{k} \cdot c_{i j}^{2}+\sum_{(i, j) \in L} y_{i j}^{k} \cdot d_{i j}^{2}+\sum_{b \in B:(i, b) \in L} \lambda_{b}^{2} \cdot y_{i b}^{k}\right)
\end{aligned}
$$

This model includes constraints (M2-2)-(M2-16) and (M2-18)-(M2-20) (see Model 2); additionally the following new constraints are added.

$$
\begin{aligned}
\sum_{w=1}^{W} x_{i j}^{w} & =0, \quad \forall(i, j) \in A_{R 2} \\
\sum_{k=W+1}^{\tau} x_{i j}^{k} & =0, \quad \forall(i, j) \in A_{R 1} \\
\sum_{w=1}^{W}\left(x_{i j}^{w}+x_{j i}^{w}\right) & =0, \quad \forall(i, j) \in E_{R 2} \\
\sum_{k=W+1}^{\tau}\left(x_{i j}^{k}+x_{j i}^{k}\right) & =0, \quad \forall(i, j) \in E_{R 1}
\end{aligned}
$$

$$
\begin{aligned}
\sum_{(1, j) \in L} y_{1 j}^{1} & =1, \quad \forall j \in V \\
\sum_{(i, 1) \in L} y_{i 1}^{W} & =1, \quad \forall i \in V \\
\sum_{(1, j) \in L} y_{1 j}^{W+1} & =1, \quad \forall j \in V \\
\sum_{(i, 1) \in L} y_{i 1}^{\tau} & =1, \quad \forall i \in V
\end{aligned}
$$

Constraint (M2-17) of Model 2 was replaced by constraints (M3-10) and (M3-11).

$$
\begin{aligned}
& z_{i j}^{w} \leq Q_{1}\left(y_{i j}^{w}+x_{i j}^{w}\right), \quad \forall(i, j) \in L ; w=1, \ldots, W \quad \\
& z_{i j}^{k} \leq Q_{2}\left(y_{i j}^{k}+x_{i j}^{k}\right), \quad \forall(i, j) \in L ; k=W+1, \ldots, \tau
\end{aligned}
$$

Vehicle $T_{1}$ cannot serve certain arcs (M3-2) and edges (M3-4). The same thing applies for vehicle $\mathrm{T}_{2}$ ((M3-3) and (M3-5)). The capacities of vehicles $T_{1}$ and $T_{2}$ must be respected ((M3-10) and (M3-11)).

\section{COMPUTATIONAL RESULTS}

The computational results are divided into two parts, group 1 , based on instances adapted from the literature, and group 2, which is dedicated to the Monção waste collection case. These results were obtained using the solver CPLEX 12.5 (IBM) in an Intel Core2 Duo $3.00 \mathrm{GHz}$ computer with 8.00 GB of RAM.

\subsection{Group 1-Instances from the literature}

These computational results were gathered for the case of one vehicle and multiple disposal sites (described in Model 2). A good possibility to test and evaluate Model 2 is using the following (two) sets of instances from the literature, after an adequate adaptation. The first set is the $G D B_{i}, i=1,2, \ldots, 23$ instances from [17]. The second set is the one composed of mvalxy, $x=1,2, \ldots, 10, y=A, B, C,(D)$ instances used for instance by [7]. To adapt the above mentioned instances, some vertices, edges, and arcs were added to each original instance. The vertices consist of a vertex " 1 " that corresponds to the garage and a further 2,3 , or 4 new vertices that represent, respectively, 2, 3, or 4 disposal sites. Certain edges and arcs were also added to maintain one connected component in each instance.

The following notation is used in the columns of the tables:

- ID: instance;

- $\tau$ : number of trips;

- $\sharp V$ : number of vertices (depot + landfills + ordinary vertices);

- $\sharp R$ : number of required links;

- $\sharp \bar{R}$ : number of nonrequired links;

- $Q$ : capacity of the vehicle;

- $\sharp B$ : number of disposal sites; 
TABLE 1. Instances $G D B_{i-} L M L$ and $G D B_{15} \_M L_{i}$.

\begin{tabular}{|c|c|c|c|c|c|c|c|c|c|c|}
\hline ID & $\tau$ & $\sharp V$ & $\sharp R$ & $\sharp \bar{R}$ & Q & $\sharp \mathrm{B}$ & $\operatorname{Max}$ & $\operatorname{Gap}(\%)$ & Time (sec) & OF \\
\hline$G D B_{1 \_} L M L$ & 6 & 15 & 22 & 6 & 5 & 2 & {$[3,3]$} & - & 70,71 & $310^{*}$ \\
\hline$G D B_{2} \_L M L$ & 7 & 15 & 26 & 6 & 5 & 2 & {$[4,4]$} & - & 258,37 & $347^{*}$ \\
\hline$G D B_{3} \_L M L$ & 6 & 15 & 22 & 6 & 5 & 2 & {$[3,3]$} & - & 7,96 & $286^{*}$ \\
\hline$G D B_{4} \_L M L$ & 5 & 14 & 19 & 6 & 5 & 2 & {$[3,3]$} & - & 17,25 & $293^{*}$ \\
\hline$G D B_{5} L L L$ & 7 & 16 & 26 & 6 & 5 & 2 & {$[3,3]$} & - & 133,41 & $376^{*}$ \\
\hline$G D B_{6 \_} L M L$ & 6 & 15 & 22 & 6 & 5 & 2 & {$[3,3]$} & - & 680,23 & $317^{*}$ \\
\hline$G D B_{7} L L M L$ & 6 & 15 & 22 & 6 & 5 & 2 & {$[3,3]$} & - & 67,55 & 329* \\
\hline$G D B_{8} \_L M L$ & 11 & 30 & 46 & 6 & 27 & 2 & {$[5,5]$} & 10,91 & $3 \mathrm{~h}$ & 364 \\
\hline$G D B_{9} \_L L$ & 11 & 30 & 51 & 6 & 27 & 2 & {$[5,5]$} & 11,14 & $3 \mathrm{~h}$ & 353 \\
\hline$G D B_{10} \_L M L$ & 5 & 15 & 25 & 6 & 10 & 2 & {$[2,2]$} & - & 3,93 & $296^{*}$ \\
\hline$G D B_{11 \_} L M L$ & 6 & 25 & 45 & 6 & 50 & 2 & {$[3,3]$} & 0,24 & $3 \mathrm{~h}$ & 414 \\
\hline$G D B_{12} \_L M L$ & 8 & 16 & 23 & 6 & 35 & 2 & {$[4,4]$} & 7,06 & $3 \mathrm{~h}$ & 472 \\
\hline$G D B_{13} \_L M L$ & 7 & 13 & 28 & 6 & 41 & 2 & {$[4,4]$} & 0,36 & $3 \mathrm{~h}$ & 553 \\
\hline$G D B_{14} \_L M L$ & 6 & 10 & 21 & 6 & 21 & 2 & {$[3,3]$} & - & 22,95 & $123^{*}$ \\
\hline$G D B_{15} \_L M L$ & 5 & 10 & 21 & 6 & 37 & 2 & {$[2,2]$} & - & 2,53 & $\mathbf{7 8}^{*}$ \\
\hline$G D B_{16} L M L$ & 6 & 11 & 28 & 6 & 24 & 2 & {$[3,3]$} & - & 0,42 & $149^{*}$ \\
\hline$G D B_{17} \_L M L$ & 6 & 11 & 28 & 6 & 41 & 2 & {$[3,3]$} & - & 2,06 & $115^{*}$ \\
\hline$G D B_{18 \_} L M L$ & 6 & 12 & 36 & 6 & 37 & 2 & {$[3,3]$} & - & 26,02 & $187^{*}$ \\
\hline$G D B_{19} \_L M L$ & 4 & 11 & 11 & 6 & 27 & 2 & {$[2,2]$} & - & 0,12 & $77^{*}$ \\
\hline$G D B_{20} \_L M L$ & 5 & 14 & 22 & 6 & 27 & 2 & {$[2,2]$} & - & 8,19 & $150^{*}$ \\
\hline$G D B_{21} \_L M L$ & 7 & 14 & 33 & 6 & 27 & 2 & {$[3,3]$} & - & 71,54 & 184* \\
\hline$G D B_{22} \_L M L$ & 9 & 14 & 44 & 6 & 27 & 2 & {$[5,5]$} & - & 228,40 & $232^{*}$ \\
\hline$G D B_{23} \_L M L$ & 11 & 14 & 55 & 6 & 27 & 2 & {$[6,6]$} & 3,25 & $3 \mathrm{~h}$ & 277 \\
\hline$G D B_{15 \_} L M L 1$ & 5 & 10 & 21 & 6 & 37 & 2 & {$[1,4]$} & - & 0,14 & $\mathbf{7 8}^{*}$ \\
\hline$G D B_{15} \_L M L 2$ & 5 & 11 & 21 & 13 & 37 & 3 & {$[2,2,2]$} & - & 0,34 & $77^{*}$ \\
\hline$G D B_{15} \_L M L 3$ & 5 & 12 & 21 & 15 & 37 & 4 & {$[1,1,1,2]$} & - & 0,72 & $71^{*}$ \\
\hline
\end{tabular}

- $\left[\operatorname{Max} l_{i}\right]_{i=1, \ldots . \sharp B}$ : maximum number of disposals on disposal sites i;

- $\operatorname{Gap}(\%)$ : relative gap obtained with CPLEX 12.5;

- Time(sec): computational time in seconds (" $3 \mathrm{~h}$ "= best solution after $3 \mathrm{~h}$ running);

- OF: objective function [(optimum value (bold*) or upper bound].

Table 1 presents a set of 23 instances $G D B_{i_{-}} L M L, i=$ $1, \ldots, 23$ and three more instances developed from $G D B_{15} \_M L$ with 2,3 , or 4 disposal sites (available in http:// www.inescporto.pt/ amr/Limited_Multi_Landfills).

(The original instances are available in http:/www.uv.es/ belengue/carp.html).

Using the same transformation, Table 2 shows the results of 34 instances, mvalXY_LML, $X=1,2, \ldots, 10$ and $Y=A$, $B, C$ (and, in some cases, also $Y=D$ ), adapted from the literature. These 34 instances only have two disposal sites. The maximum number of disposals for each site is always $T-1 \mathrm{Max}=\left[\operatorname{Max} l_{i}\right]_{i=1,2}=[T-1, T-1]$, meaning that each of them could absorb all disposals (instances available in http://www.inescporto.pt/ amr/Limited_Multi_Landfills).

(The original instances, called mval, are available in http://www.uv.es/belengue/mcarp/index.html)

Some other instances were generated ( $m v a l 1 Y \_L M L_{i}$, com $Y=A, B, C$ e $i=1,2)$ using instance mval1Y_LML as foundation. The modifications introduced consist of the number of disposal sites and/or the maximum number of disposals at each point (available in http://www. inescporto.pt/ amr/Limited_Multi_Landfills).

Table 3 presents the results obtained.
This last set of instances is the result of a few changes to the number of disposal sites.

Tables 1-3 show that the optimal solutions were achieved in 20 of 26 instances GDBi_LML and in 29 of 43 instances mvali_LML, within $3 \mathrm{~h}$ of computational time. Notice that while $G D B_{i_{-}} L M L$ instances are basically constituted by edges in the case of mvalXY_LML instances arcs are in a larger number than the edges.

The computational results also indicate that there are relations between "trips" and "gaps": the gap is directly proportional to the number of trips. This is due to the fact that smaller capacity vehicles are filled quickly, having to resort more often to the disposal sites. Furthermore, optimal solutions were obtained whenever the number of trips does not exceed 5; this is a good result because, in a practical situation, the number of disposals is 4 or less.

\subsection{Group 2-Instances from Monção Waste Collection}

The results of Group 2 are related to Monção and they are based on real data. Figure 1 shows that region and, in particular, the locations of the garage and of a landfill and a transfer station.

It should be emphasized that a sectorization phase takes place before the route planning process. It is essential to make a partition of the county to know which part (sector) will be served each day/or per route. It is important to highlight that the periodicity of the collection is not equal for all the boroughs in the county.

The following explanations should be taken into consideration: 
TABLE 2. Characteristics and results for instances mvalXY_LML.

\begin{tabular}{|c|c|c|c|c|c|c|c|c|}
\hline ID & $\tau$ & $\sharp V$ & $\sharp E_{R}$ & $\sharp A_{R}$ & Q & $\operatorname{Gap}(\%)$ & Time (sec) & OF \\
\hline mval1A_LML & 3 & 27 & 20 & 35 & 200 & - & 0,25 & 239* \\
\hline mval1B_LML & 4 & 27 & 13 & 38 & 120 & - & 0,44 & $274^{*}$ \\
\hline mval1C_LML & 9 & 27 & 17 & 36 & 45 & 10,50 & $3 \mathrm{~h}$ & 379 \\
\hline mval2A_LML & 3 & 27 & 16 & 28 & 180 & - & 0,08 & 332* \\
\hline mval2B_LML & 4 & 27 & 12 & 40 & 120 & - & 1,37 & $395^{*}$ \\
\hline mval2C_LML & 9 & 27 & 14 & 35 & 40 & 8,60 & $3 \mathrm{~h}$ & 508 \\
\hline mval3A_LML & 3 & 27 & 15 & 33 & 80 & - & 0,09 & $128^{*}$ \\
\hline$m v a l 3 B \_L M L$ & 4 & 27 & 16 & 29 & 50 & - & 0,47 & $158^{*}$ \\
\hline mval3C_LML & 8 & 27 & 18 & 25 & 20 & 4,99 & $3 \mathrm{~h}$ & 188 \\
\hline mval4A_LML & 4 & 44 & 26 & 69 & 225 & - & 1,64 & 597* \\
\hline mval4B_LML & 5 & 44 & 19 & 83 & 170 & - & 41,86 & $665^{*}$ \\
\hline mval4C_LML & 6 & 44 & 21 & 82 & 130 & - & 23,74 & $645^{*}$ \\
\hline mval4D_LML & 10 & 44 & 21 & 83 & 75 & 6,32 & $3 \mathrm{~h}$ & 748 \\
\hline mval5A_LML & 4 & 37 & 22 & 74 & 220 & - & 4,88 & $625^{*}$ \\
\hline$m v a l 5 B \_L M L$ & 5 & 37 & 35 & 56 & 165 & - & 34,35 & $613^{*}$ \\
\hline mval5C_LML & 6 & 37 & 17 & 81 & 130 & - & 3,96 & $685^{*}$ \\
\hline mval5D_LML & 10 & 37 & 29 & 63 & 75 & 4,38 & $3 \mathrm{~h}$ & 666 \\
\hline mval6A_LML & 4 & 34 & 22 & 47 & 170 & - & 1,44 & $342^{*}$ \\
\hline mval6B_LML & 5 & 34 & 22 & 44 & 120 & - & 11,87 & $337^{*}$ \\
\hline mval6C_LML & 11 & 34 & 23 & 45 & 50 & 9,21 & $3 \mathrm{~h}$ & 421 \\
\hline mval7A_LML & 4 & 43 & 36 & 50 & 200 & - & 1,45 & $384^{*}$ \\
\hline$m v a l 7 B \_L M L$ & 5 & 43 & 25 & 66 & 150 & - & 3,71 & $432^{*}$ \\
\hline mval7C_LML & 10 & 43 & 28 & 62 & 65 & 6,51 & $3 \mathrm{~h}$ & 466 \\
\hline mval8A_LML & 4 & 33 & 20 & 76 & 200 & - & 1,12 & $595^{*}$ \\
\hline mval8B_LML & 5 & 33 & 27 & 64 & 150 & - & 10,98 & $545^{*}$ \\
\hline mval8C_LML & 10 & 33 & 28 & 55 & 65 & 9,02 & $3 \mathrm{~h}$ & 709 \\
\hline mval9A_LML & 4 & 53 & 32 & 100 & 235 & - & 10,30 & $476^{*}$ \\
\hline mval9B_LML & 5 & 53 & 44 & 76 & 175 & - & 5778,82 & $488^{*}$ \\
\hline mval9C_LML & 6 & 53 & 42 & 83 & 140 & 2,05 & $3 \mathrm{~h}$ & 463 \\
\hline mval9D_LML & 11 & 53 & 38 & 93 & 70 & 11,81 & $3 \mathrm{~h}$ & 654 \\
\hline mval10A_LML & 4 & 53 & 32 & 106 & 250 & - & 2,07 & $646^{*}$ \\
\hline mval10B_LML & 5 & 53 & 33 & 101 & 190 & - & 19,59 & $667^{*}$ \\
\hline mval10C_LML & 6 & 53 & 36 & 100 & 150 & - & 64,05 & $642^{*}$ \\
\hline mval10D_LML & 11 & 53 & 42 & 87 & 75 & 13,09 & $3 \mathrm{~h}$ & 739 \\
\hline
\end{tabular}

TABLE 3. Characteristics and results for instances $m v a l 1 Y_{-} L M L_{i}$.

\begin{tabular}{|c|c|c|c|c|c|c|c|c|c|}
\hline ID & $\sharp V$ & $\sharp E_{R}$ & $\sharp A_{R}$ & Q & $\sharp \mathrm{B}$ & Max & $\operatorname{Gap}(\%)$ & Time (sec) & $\mathrm{OF}$ \\
\hline mval1 $1 A_{1 \_L M L}$ & 27 & 20 & 35 & 200 & 2 & {$[2,2]$} & - & 0,25 & 239* \\
\hline mval1A_LML $L_{1}$ & 27 & 20 & 35 & 200 & 2 & {$[1,1]$} & - & 0,25 & 239* \\
\hline mval1A_LML $L_{2}$ & 28 & 20 & 35 & 200 & 3 & {$[2,2,2]$} & - & 0,22 & $237^{*}$ \\
\hline mval1B_LML & 27 & 13 & 38 & 120 & 2 & {$[3,3]$} & - & 0,44 & $274^{*}$ \\
\hline$m v a l 1 B \_L M L_{1}$ & 28 & 13 & 38 & 120 & 3 & {$[2,1,2]$} & - & 0,45 & $272 *$ \\
\hline$m v a l 1 B \_L M L_{2}$ & 28 & 13 & 38 & 120 & 3 & {$[1,1,3]$} & - & 0,20 & $273^{*}$ \\
\hline mval1C_LML & 27 & 17 & 36 & 45 & 2 & {$[8,8]$} & 10,52 & $3 \mathrm{~h}$ & 379 \\
\hline mval1C_LML $L$ & 28 & 17 & 36 & 45 & 3 & {$[4,3,3]$} & 10,13 & $3 \mathrm{~h}$ & 341 \\
\hline mval1C_LML $L$ & 29 & 17 & 36 & 45 & 4 & {$[3,3,3,3]$} & 12,20 & $3 \mathrm{~h}$ & 367 \\
\hline
\end{tabular}

- instances "AltoMinhoX" represent Monção waste collection;

- a link (edge or arc) can be a street or a set of streets (reducing the amount of data);

- $\sharp R$ - the number of required edges and arcs, which means streets with containers;

- $\sharp \bar{R}$ - number of non required edges and arcs, streets without containers;

- if in a street there is more than one container, a false container with a capacity that is equal to the sum of the capacities of all containers will represent the demand on that street;

- the objective function (OF) (represented by the time spent in the collection process, in seconds) depends on several factors: the length of the street, the number and type of containers, the time to collect each type of container and the average speed of the vehicle.

Information about waste collection instances in Monção is available in http://www.inescporto.pt/ amr/Limited Multi_Landfills.

Table 4 shows some results obtained from the case of Monção, described in section 3, using one vehicle. The estimated time to empty is equal to $20 \mathrm{~min}$ for both disposal sites. 


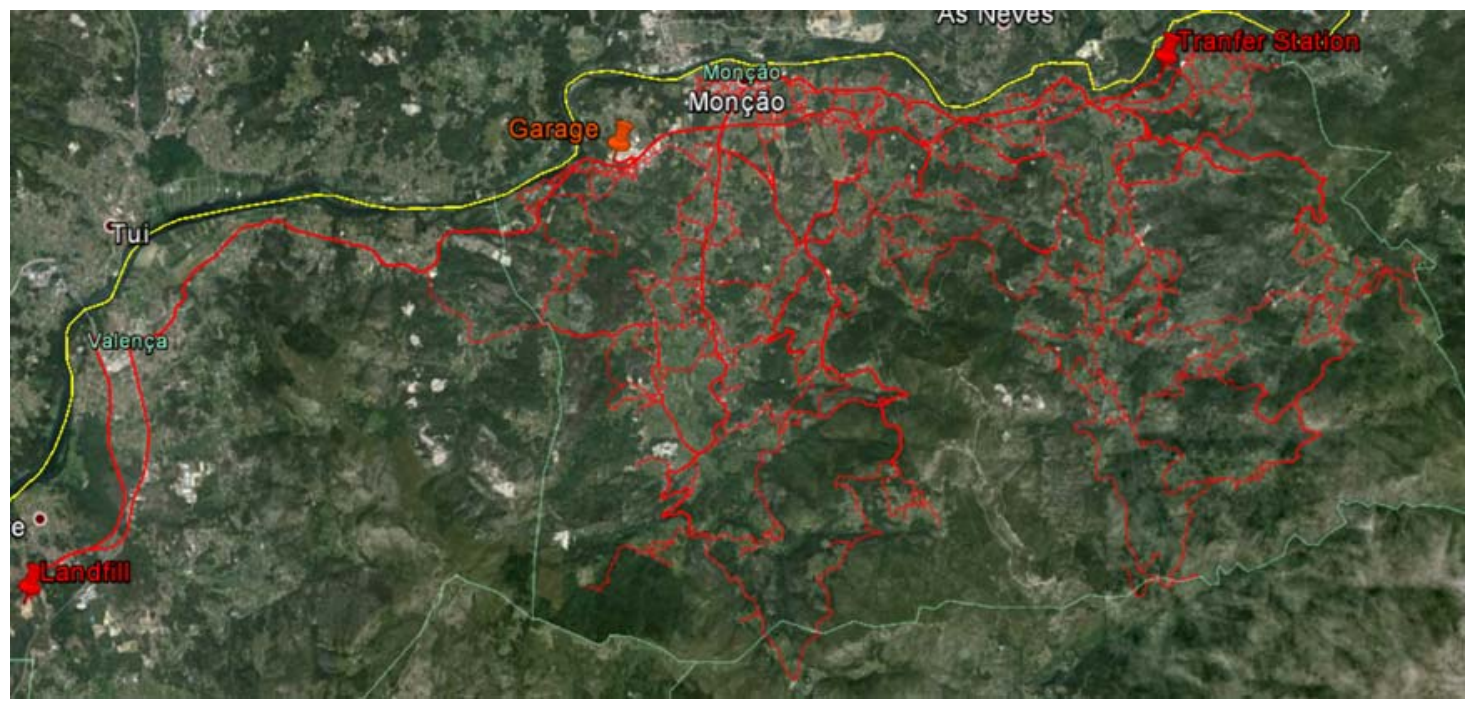

FIG. 1. The region of Monção by Google Earth. [Color figure can be viewed in the online issue, which is available at wileyonlinelibrary.com.].

TABLE 4. Characteristics and results in Monção waste collection (one vehicle).

\begin{tabular}{crrrrrrrrr}
\hline ID & $\tau$ & $\sharp V$ & $\sharp R$ & $\sharp \bar{R}$ & $\mathrm{Q}$ & $\sharp B$ & Max & Time (sec) & Gap (\%) \\
\hline AltoMinhoA & 4 & 49 & 31 & 31 & 16 & 2 & {$[1,3]$} & 12,82 & - \\
AltoMinhoB & 4 & 60 & 59 & 17 & 16 & 2 & {$[1,3]$} & 2,20 & - \\
AltoMinhoC & 4 & 182 & 144 & 121 & 16 & 2 & {$[3,1]$} & 456,79 & - \\
AltoMinhoD & 4 & 200 & 165 & 124 & 16 & 2 & {$[3,1]$} & 2719,66 & $\mathbf{2 9 ~ 9 8 2}^{*}$ \\
\hline
\end{tabular}

TABLE 5. Characteristics and results in the Monção waste collection (two vehicles).

\begin{tabular}{cccccccccc}
\hline ID & $W, K$ & $\sharp V$ & $\sharp R$ & $\sharp \bar{R}$ & $\mathrm{Q}$ & $\sharp B$ & Max & Time (sec) & Gap (\%) \\
\hline AltoMinho2C & 3,4 & 183 & 144 & 121 & 16,7 & 2 & {$[5,1]$} & $3 \mathrm{~h}$ & 0.02 \\
AltoMinho2D & 3,3 & 200 & 165 & 124 & 16,7 & 2 & {$[5,1]$} & 4849,88 & 41240 \\
\hline
\end{tabular}

With regard to the OF, representing the duration of the complete route, the values are very high for a working day, especially for instances AltoMinhoC and AltoMinhoD. Then, a second smaller vehicle $\left(7 \mathrm{~m}^{3}\right)$ is added "to help" the first. This small vehicle cannot collect all kind of containers.

These new results for the selected instances of Monção, now using two vehicles, are available in Table 5. $W$ and $K$ denote the number of trips allowed for vehicles with capacities 16 and $7 \mathrm{~m}^{3}$, respectively. As previously mentioned, there are some streets with large containers which must be served by the $16 \mathrm{~m}^{3}$ vehicle (the one with a crane). The estimated time to empty is, in this case, independent of the disposal sites, although it differs for these two vehicles. The vehicle with the crane takes about $20 \mathrm{~min}$ and the smallest takes about $10 \mathrm{~min}$.

Instances from the case of Monção were solved using this procedure. AltoMinhoX instances assume one vehicle only, while AltoMinho $2 X$ instances assume two vehicles. The computational results show that the proposed mathematical modeling approach can produce realistic and effective solutions to the waste collection routing problem in Monção.

The fact that the main disposal site is more than $25 \mathrm{~km}$ away and the second one, the transfer station, even if closer, has some limitations concerning the number of disposals per day, which poses special difficulties for the company dealing with the waste collection. The company must maintain a schedule, ensuring that a number of containers are collected every day, while other containers, depending on the amount of waste produced, are collected two or three times a week. The solution presented in this paper is considered an efficient and realistic alternative to the (largely manual) procedure currently in use to ensure an effective response to the waste collection problem.

Figure 2 illustrates another case study with 149 vertices, 106 required links, and 114 nonrequired links. Landf2 represents the transfer station. The data are available in www.inescporto.pt/ amr/Limited_Multi_Landfills/RealCase /2Vehicles/S1_2Veic_Cenario2.txt.

The solution is optimal, $37402 \mathrm{sec}$, and it was obtained in 1341, $25 \mathrm{sec}$. The network presented corresponds to the region in blue which is part of the municipality of Monção shown in Figure 1. The red lines and gray lines represent, respectively, the paths of the smaller and of the larger vehicles. Distances between vertices do not correspond to real distances. 


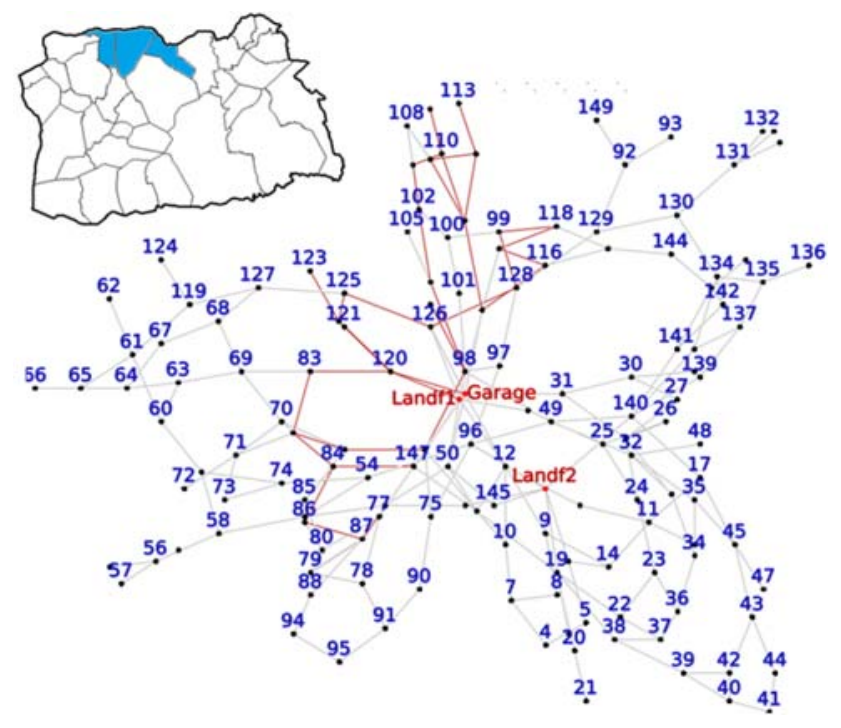

FIG. 2. Paths of the smaller vehicle (red) and of the larger vehicle (gray) and two disposal sites. [Color figure can be viewed in the online issue, which is available at wileyonlinelibrary.com.]

\section{CONCLUSION}

This article considered a real-life waste collection problem (in Monção, Portugal) involving multiple disposal sites, some with a limited number of disposals per day, and using different vehicles. Three new models, representing three different situations, were proposed and tested. They also provide a contribution to the CARP literature, due to their additional features related to heterogeneous fleet and limited multiple landfills. Moreover, they have a close connection to the practical situation described and they may be applied in other contexts. Computational results were also included based on instances adapted from the literature and based on the case of Monção. Some conclusions were drawn, namely that optimal solutions are reached whenever the number of trips does not exceed 5. This is relevant for the practical case, since it is not normal that a vehicle empties more than three or four times, which corresponds to four or five trips, respectively. The outcome of the tests also show the effectiveness of integrating sectorization and routing to solve real waste collection problems.

\section{ACKNOWLEDGMENT}

The authors also wish to thank two anonymous referees for their helpful comments and suggestions.

\section{REFERENCES}

[1] A. Amaya, A. Langevin, and M. Trépanier, The capacitated arc routing problem with refill points, Operations Research Letters 35 (2007), 45-53.

[2] S.K. Amponsah and S. Salhi, The investigation of a class of capacitated arc routing problems: The collection of garbage in developing countries, Waste Manag 24 (2004), 711-721.
[3] A.A. Assad and B.L. Golden, "Arc routing methods and applications," In Network Routing, Vol. 8 of Handbooks in Operations Research and Management Science, C.M.M.O. Ball, T.L. Magnanti, and G. Nemhauser (Eds.), Elsevier, 1995, pp. 375-483.

[4] S. Baptista, R.C. Oliveira, and E. Zúquete, A periodic vehicle routing case study, Eur J Oper Res 139 (2002), 220-229.

[5] J. Bautista, E. Fernández, and J. Pereira, Solving an urban waste collection problem using ants heuristics, Comput Oper Res 35 (2008), 3020-3033.

[6] J.M. Belenguer and E. Benavent, The capacitated arc routing problem: Valid inequalities and facets, Comput Optim Appl 10 (1998), 165-187.

[7] J.-M. Belenguer, E. Benavent, P. Lacomme, and C. Prins, Lower and upper bounds for the mixed capacitated arc routing problem, Comput Oper Res 33 (2006), 3363-3383.

[8] F. Chu, N. Labadi, and C. Prins, A scatter search for the periodic capacitated arc routing problem, Eur J Oper Res 169 (2006), 586-605.

[9] A. Corberán and C. Prins, Recent results on arc routing problems: An annotated bibliography, Networks 56 (2010), 50-69.

[10] A. Del Pia and C. Filippi, A variable neighborhood descent algorithm for a real waste collection problem with mobile depots, Int Trans Oper Res 13 (2006), 125-141.

[11] H. A. Eiselt and V. Marianov, A bi-objective model for the location of landfills for municipal solid waste, Eur J Oper Res 235 (2014), 187-194.

[12] H.A. Eiselt, M. Gendreau, and G. Laporte, Arc routing problems (PartI): The chinese postman problem, Oper Res 43 (1995a), 231-242.

[13] H.A. Eiselt, M. Gendreau, and G. Laporte, Arc routing problems (PartII): The rural postman problem, Oper Res 43 (1995b), 399-414.

[14] G. Fleury, P. Lacomme, C. Prins, G. Raidl, S. Cagnoni, J. Branke, D. Corne, R. Drechsler, Y. Jin, C. Johnson, P. Machado, E. Marchiori, F. Rothlauf, G. Smith, and G. Squillero, (Eds.). Evolutionary Algorithms for Stochastic Arc Routing Problems. Applications of Evolutionary Computing, Springer Berlin Heidelberg 3005 (2004), 501-512.

[15] G. Ghiani, G. Improta, and G. Laporte, The capacitated arc routing problem with intermediate facilities, Networks 37 (2001), 134-143.

[16] B. Golden and R. Wong, Capacitated arc routing problem, Networks 11 (1981), 305-315.

[17] B. Golden, J. Dearmon, and E. Baker, Computational experiments with algorithms for a class of routing problems, Comput Oper Res 10 (1983), 47-59.

[18] L. Gouveia, M.C. Mourão, and L.S. Pinto, Lower bounds for the mixed capacitated arc routing problem, Comput Oper Res 37 (2010), 692-699.

[19] S. Hanafi, A. Freville, and P. Vaca, Municipal solid waste collection: An effective data structure for solving the sectorization problem with local search methods, INFOR 37 (1999), 236-254

[20] IBM, CPLEX 12.5, Available at:http://www-01.ibm.com/ software/integration/optimization/cplex-optimization-studio/ (2012), Accessed on September 2013. 
[21] B.-I. Kim, S. Kim, and S. Sahoo, Waste collection vehicle routing problem with time windows, Comput Oper Res 33 (2006), 3624-3642.

[22] T. Kulcar, Optimizing solid waste collection in brussels, Eur J Oper Res 90 (1996), 71-77.

[23] P. Lacomme, C. Prins, and W. Ramdane-Chérif, "Evolutionary algorithms for multiperiod arc routing problems," Proc. of the 9th Int. Conf. on Information Processing and Management of Uncertainty in Knowledge-Based systems (IPMU 2002), ESIAUniversity of Savoie (2002), 845-852.

[24] P. Lacomme, C. Prins, and M. Sevaux, "Multiobjective capacitated arc routing problem," In Evolutionary Multi-Criterion Optimization, Vol. 2632/2003, Springer Berlin, Heidelberg, 2003.

[25] P. Lacomme, C. Prins, and W. Ramdane-Chérif, Competitive memetic algorithms for arc routing problems, Ann Oper Res 131 (2004), 159-185.

[26] P. Lacomme, C. Prins, and M. Sevaux, A genetic algorithm for a bi-objective capacitated arc routing problem, Comput Oper Res 33 (2006), 3473-3493.

[27] A.N. Letchford and A. Oukil, Exploiting sparsity in pricing routines for the capacitated arc routing problem, Comput Oper Res 36 (2009), 2320-2327.

[28] H. Longo, M.P. Aragão, and E. Uchoa, Solving capacitated arc routing problems a transformation to the cvrp, Comput Oper Res 33 (2006), 1823-1837.

[29] J.W. Male and J. C. Liebman, Districting and routing for solid waste collection, J Environ Eng Div 104 (1978), 1-14.

[30] M.C. Mourão and M.T. Almeida, Lower-bouding and heuristic methods for a refuse collection vehicle routing problem, Eur J Oper Res 121 (2000), 420-434.
[31] M.C. Mourão, A.C. Nunes, and C. Prins, Heuristic methods for the sectoring arc routing problem, Eur J Oper Res 196 (2009), 856-868.

[32] L. Muyldermans, D. Cattrysse, and D. Van Oudheusden, District design for arc-routing applications, J Oper Res Soc 54 (2003), 1209-1221.

[33] T. Nuortio, J. Kytöjoki, H. Niska, and Bräysy, Improved route planning and scheduling of waste collection and transport, Expert Syst Appl 30 (2006), 223-232.

[34] M. Polacek, K.F. Doerner, R.F. Hartl, and V. Maniezzo, A variable neighborhood for the capacitated arc routing problem with intermediate facilities, J Heuristics 14 (2008), 405-423.

[35] A. M. Rodrigues and J. S. Ferreira, An electromagnetism based approach to sectorization, INESC TEC/ FEUP (in press).

[36] S. Sahoo, S. Kim, B.-I. Kim, B. Kraas, and A. Popov, Jr . Routing optimization for waste management, Interfaces 35 (2005), 24-36.

[37] M. Salazar-Aguilar, A. Langevin, and G. Laporte, The synchronized arc and node routing problem: Application to road marking, Comput Oper Res 40 (2013), 17081715.

[38] M. Tagmouti, M. Gendreau, and J.-Y. Potvin, Arc routing problems with time-dependent service costs, Eur J Oper Res 181 (2007), 30-39.

[39] S. Wøhlk, A decade of capacitated arc routing. In: B.L. Golden, S. Raghavan, and E.A. Wasil (eds.) The Vehicle Routing Problem: Latest Advances and New Challenges, Springer Science+Business Media, Springer, Boston (2008), 29-48. 